\title{
Capture of the eyes by relevant and irrelevant onsets
}

\author{
Manon Mulckhuyse $\cdot$ Wieske van Zoest $\cdot$ Jan Theeuwes
}

Received: 22 June 2007 / Accepted: 19 November 2007 / Published online: 5 December 2007

(C) The Author(s) 2007

\begin{abstract}
During early visual processing the eyes can be captured by salient visual information in the environment. Whether a salient stimulus captures the eyes in a purely automatic, bottom-up fashion or whether capture is contingent on task demands is still under debate. In the first experiment, we manipulated the relevance of a salient onset distractor. The onset distractor could either be similar or dissimilar to the target. Error saccade latency distributions showed that early in time, oculomotor capture was driven purely bottom-up irrespective of distractor similarity. Later in time, top-down information became available resulting in contingent capture. In the second experiment, we manipulated the saliency information at the target location. A salient onset stimulus could be presented either at the target or at a non-target location. The latency distributions of error and correct saccades had a similar time-course as those observed in the first experiment. Initially, the distributions overlapped but later in time task-relevant information decelerated the oculomotor system. The present findings reveal the interaction between bottom-up and topdown processes in oculomotor behavior. We conclude that the task relevance of a salient event is not crucial for capture of the eyes to occur. Moreover, task-relevant information may integrate with saliency information to initiate saccades, but only later in time.
\end{abstract}

Keywords Eye-movement - Capture - Attention ·

Time-course

M. Mulckhuyse $(\square) \cdot$ W. van Zoest $\cdot$ J. Theeuwes

Cognitive Psychology, Vrije Universiteit,

Van der Boechorststraat 1, 1081 BT Amsterdam,

The Netherlands

e-mail: M.Mulckhuyse@psy.vu.nl

\section{Introduction}

Imagine walking along a path next to a lake. You are searching for a particular red flower that grows only along the waterfront. While searching, all red flowers will probably attract your attention. Your eyes will fixate on the red flowers while at the same time you ignore other colorful flowers. You can ignore the other flowers because they do not share the feature you are set to look for; the color red. However, if suddenly a fish jumps up out of the water your attention and your eyes are captured automatically by this event even though it has nothing to do with your current goal. These two examples illustrate the balance between top-down and bottom-up attentional and oculomotor capture. The former is induced by the contingency of top-down goals and the latter is induced by the salience information in the environment. However, whether a salient event captures attention in a purely automatic, bottom-up fashion or whether capture is always contingent on task demands is still under debate. The aim of this study was to investigate the role of these bottom-up and top-down selection processes in a visual search task using oculomotor capture as the dependent measure.

Attentional capture is commonly measured by presenting an additional irrelevant—but salient distractor during visual search (e.g., Theeuwes 1992, 1994a, b, 1996; Yantis and Jonides 1984, 1990). Typically, the presence of this distractor slows manual responses to the target. Results from these studies led to a bottom-up capture hypothesis proposed by Theeuwes (1992, 1994a, 2004). This view states that during early visual processing, bottom-up saliency signals in the environment can cause an involuntary shift of spatial attention that cannot be overridden by attentional top-down goals. Bacon and Egeth (1994), however, proposed an alternative explanation. Not the saliency 
of the distractor, but the fact that both target and distractor were defined by a unique singleton in the display, caused a shift of attention. They hypothesized that participants adopted a top-down search strategy for singletons and therefore any singleton would capture attention (but see Theeuwes 2004). This view is consistent with the contingent capture hypothesis of Folk and colleagues (e.g., Folk et al. 1992, 1994). This hypothesis states that even at the early stage in visual processing, top-down goals induced by task demands are crucial for attentional capture (e.g., Folk et al. 1992, 1994). In these studies, a distractor cue, that can be similar or dissimilar to the to search for target, is presented preceding the target display to measure attentional capture. Results showed that a distractor cue caused a shift of spatial attention only if distractor and target were defined by the same feature properties.

In the oculomotor domain, studies on saliency and contingency have provided additional insights regarding the mechanism underlying attentional capture (e.g., Ludwig and Gilchrist 2002, 2003a; van Zoest and Donk 2006). For example, in a recent study by van Zoest and Donk (2006), subjects had to make a speeded saccade to a vertical lineelement (i.e., the target) that was presented in a display full of background line-elements. These background line-elements all had the same contrasting orientation relative to the target. An irrelevant singleton (i.e., distractor) that differed in orientation from the homogeneous group of background elements was presented in each trial. They manipulated the saliency of this distractor by increasing or decreasing the orientation contrast to the surrounding background line-elements. In addition, the similarity of the distractor to the target was manipulated by making the orientation of the distractor line-element more or less similar to the target. Results showed that the short-latency saccades went more often to the salient distractor while longlatency saccades were not affected by distractor saliency. In contrast, the short-latency saccades were not affected by target-distractor similarity while long-latency saccades went more often to the similar distractor. Moreover, no interaction was found between distractor saliency and target-distractor similarity suggesting that saccades driven by saliency were independent of saccades driven by top-down goals. Although this study shows that short-latency saccades are driven by bottom-up saliency, consistent with a bottom-up capture view, the results are not in line with the idea of a contingency between bottom-up and top-down control. Based on their results, van Zoest and Donk suggest that saliency and top-down processes operate in different and independent time-windows and do not integrate to guide behavior.

In the studies by Ludwig and Gilchrist (2002, 2003a), subjects had to make a speeded saccade to one of four possible target locations while ignoring a distractor at two other possible locations. The distractor could be similar (i.e., identical) or dissimilar to the target. In addition, the saliency of the similar or dissimilar distractor type was manipulated by presenting it either as an abrupt onset or as a color change. Relative to baseline, in which no distractor was present, a dissimilar distractor, regardless whether it was more or less salient, had no effect on saccade latencies to the target and hardly captured the eyes. On the contrary, the similar distractor, whether it was more or less salient, increased saccade latencies to the target and could capture the eyes up to $56 \%$ of the trials. They concluded that their results are consistent with the contingent capture hypothesis (Folk et al. 1992, 1994) although in a weaker version. Ludwig and Gilchrist argued that their results are in line with a weaker version of the contingent capture hypothesis because the eyes were captured significantly more often by the more salient similar distractor (abrupt onset) than by the less salient similar distractor (color change). Furthermore, when the eyes were captured, saccade latencies were shorter to onset distractors than to no-onset distractors. Therefore, the saliency of this contingent stimulus not only affected the number of times the eyes were captured but also the latencies of these error saccades. In contrast to van Zoest and Donk (2006), Ludwig and Gilchrist argued that bottom-up saliency signals are integrated with top-down information onto a common oculomotor saliency map to determine the saliency of an item (see also Godijn and Theeuwes 2002 for a similar conclusion).

However, Ludwig and Gilchrist (2002, 2003a), based their conclusion on the results obtained with the similar distractor. In their study, the dissimilar salient distractor did not affect saccade latencies and hardly captured the eyes. This could be due to the fact that the location of the distractor was highly predictable. Moreover, unlike the design used by van Zoest and Donk (2006) the irrelevant distractor could never occur at a potential target location. Possibly, subjects in Ludwig and Gilchrist's studies could have inhibited the possible distractor locations beforehand. Indeed as shown in a study by Van der Stigchel and Theeuwes (2006) the mere expectation that a distractor will appear at a specific location can result in inhibition of that location. In this study, Van der Stigchel and Theeuwes found saccade deviations away from a location where a distractor was expected. In Ludwig and Gilchrist study, this location-based inhibition may have allowed the participants to successfully ignore the dissimilar distractor, but not the similar distractor (see also Ludwig and Gilchrist 2003b). In the present study, we used four possible distractor locations, which makes the distractor location less predictable in contrast to using two possible locations. The paradigm we used was a variant of the additional singleton paradigm in which subjects make a speeded saccade to a color singleton while a sudden onset can appear at one of the four possible locations (Godijn and Theeuwes 2002). In the 
first experiment, the saliency of the distractor (an abrupt onset) was equal in both conditions. We manipulated the contingency of the additional distractor by making the color similar (similar onset condition) or dissimilar (dissimilar onset condition) to the target. In addition, the distractor in both conditions differed in shape from the target to eliminate any other shared features. The baseline (neutral condition) consisted of trials without an onset distractor. In contrast to the original additional singleton paradigm in which the nontargets change color in an equiluminant fashion (see Theeuwes et al. 1998), we changed the color of the target in an equiluminant fashion. In a previous study, Godijn and Theeuwes (2002) showed that an equiluminant color change of the target basically gives the same results as changing all colors except the one of the target as was done in the original oculomotor capture paradigm of Theeuwes et al. (1998). Note that equiluminant color changes do not capture attention (see Theeuwes 1995). In addition, because our main interest was in the error saccades, we removed the fixation point at the moment of target onset. Fixation disengagement is known to decrease saccade latencies (e.g., Munoz et al. 2000) and short-latency saccades are associated with an increase in oculomotor capture (Godijn and Theeuwes 2002).

We expected to find an effect of distractor presence and distractor similarity on correct saccades: slower saccades on trials with an onset distractor relative to baseline and slower saccades on trials with a similar onset distractor relative to trials with a dissimilar onset distractor. Furthermore, we expected both types of onset distractors to capture the eyes automatically; yet, we predicted that this would occur only when responses are triggered very early in time. Top-down information about distractor similarity may become evident only later in time due to the contingency of the distractor on task demands.

\section{Experiment 1}

\section{Method}

\section{Participants}

Twelve paid volunteers (aged 18-24) participated in the experiment. All participants had normal or corrected to normal vision.

\section{Apparatus and design}

A Pentium IV computer with a processor speed of $2.3 \mathrm{GHz}$ controlled the timing of the events. Displays were presented on an Iiyama $21^{\prime \prime}$ SVGA monitor with a resolution of 1,024 pixels $\times 768$ pixels and a $100-\mathrm{Hz}$ refresh rate. A second computer controlled the registration of eye movement data on-line. Eye movements were registered by means of a video-based eye tracker (SR Research Ltd, Canada). The Eyelink2 system has a $500-\mathrm{Hz}$ temporal resolution and a $<0.01^{\circ}$ of gaze resolution (noise limited) and a gaze position accuracy of $<0.5^{\circ}$. The system uses an infrared videobased tracking technology to compute the pupil center and pupil size of both eyes. An infrared head mounting tracking system tracked head motion. Both the eyes were monitored, but only those data from the right eye were analyzed. Although the system compensates for head movements, the participant's head was stabilized using a chin rest. The distance between monitor and chin rest was $75 \mathrm{~cm}$. The experiment was conducted in a sound-attenuated and dimly lit room.

The experiment consisted of three blocks. One block without an onset (neutral condition), one block with a gray square onset (similar onset condition) and one block with a red square onset (dissimilar onset condition). The distractor conditions each consisted of 384 trials and the neutral condition of 32 trials. Each block started with ten practice trials. The blocks were counterbalanced between participants.

We used a variant of the oculomotor capture task (Godijn and Theeuwes 2002). All stimuli were presented on a black background. The display consisted of six filled circles, each $1.45^{\circ}$ in diameter that were equally spaced around the fixation point on an imaginary circle with a radius of $9.8^{\circ}$. The circles were presented at 1, 3, 5, 7, 9 and 11 o'clock with an angular separation of $60^{\circ}$ between the circles. In the distractor conditions a filled square, $1.3^{\circ}$ in diameter, was presented on the imaginary circle exactly between two filled circles. The stimuli could be either red $(x=0.591, y=0.333)$ or gray $(x=0.285, y=0.306)$. The red and gray colors were matched for luminance $\left(14.55 \mathrm{~cd} / \mathrm{m}^{2}\right)$.

Figure 1a shows the sequence of a trial in the similar distractor condition. Each trial began with a white plus sign. After 1,400 ms the red circles were presented for at least $500 \mathrm{~ms}$ with an additional random jitter between 0 and $200 \mathrm{~ms}$. One of the red circles changed to gray indicating the target location. Simultaneously with the color change, the fixation point disappeared and in the distractor conditions, the square was presented. The target appeared equally often at each of the four possible target locations: 1 , 5,7 or 11 o'clock. The distractor could appear at 2, 4, 8 or 10 o'clock, but always with an angular separation of $90^{\circ}$ or $150^{\circ}$ between distractor and target.

\section{Procedure}

Before the experiment started, the Eyelink2 system was calibrated. Participants had to fixate nine calibration targets that were presented randomly in a $3 \times 3$ grid across the monitor. As soon as a point was fixated, the next target was displayed on the screen. On each trial in the experiment, 


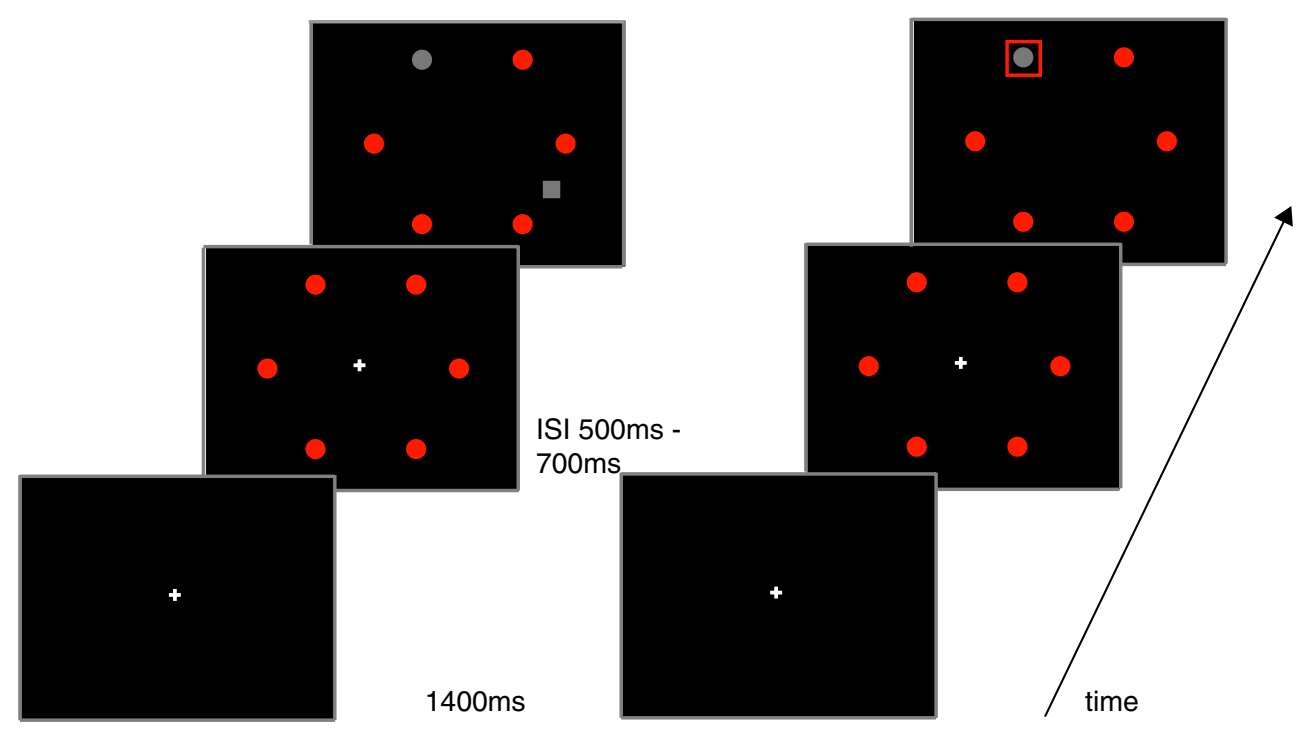

Fig. 1 a Experiment 1. From bottom to top, succession of events in a trial in the similar onset condition in which target and distractor have the same color. b Experiment 2. From bottom to top, succession of

participants were instructed to fixate the center fixation point (a cross) and to press the space bar in order to recalibrate the position of the eyes. The fixation point then changed into a plus sign as an indication that the positions of the eyes were recalibrated.

Participants were told to make a speeded saccade to the gray circle. To ensure that participants tried to be as fast as possible, we presented a warning beep if participants responded to slow (i.e., after $300 \mathrm{~ms}$ ) and excluded these slow responses from analyses. To avoid anticipation saccades the warning beep was also presented when participants responded to fast, i.e., before $50 \mathrm{~ms}$. Participants were asked to be as quick as possible and to minimize the warning beeps. In the distractor condition, feedback about their response time was displayed on the screen after every 24 th trial and in the neutral condition after every 16 th trial.

\section{Results}

Of all trials, the initial saccade latencies below $80 \mathrm{~ms}$ $(4.95 \%)$ and above $300 \mathrm{~ms}(4.72 \%)$ were excluded from analysis. The first because these are anticipation saccades and the second because these responses were to slow (see Sect. "Procedure"). The initial saccade was assigned to a target or a distractor if the endpoint of the initial saccade was within $3^{\circ}$ of the center of the target or distractor position.

Initial saccades

In the neutral condition $87 \%$ of all initial saccades went to the target. In the dissimilar onset condition $77 \%$ and in the events in a trial in the valid onset condition in which an onset is presented at target location

similar onset condition $55 \%$ of all initial saccades went to the target. A Friedman test on percentage correct saccades showed that there was a significant effect of condition $\left[\chi^{2}(2, N=12)=18.667, P<0.01\right]$.

In the dissimilar onset condition $8 \%$ of all initial saccades went to the distractor and in the similar onset condition $26 \%$ of all initial saccades went to the distractor. A two-related Wilcoxon test showed a significant effect between the two conditions $(z=3.059, N$-ties $=12, P<0.01)$.

In the neutral condition $13 \%$ of all initial saccades went to locations elsewhere than the target, in the dissimilar onset condition $15 \%$ went to locations elsewhere than the target or the distractor and in the similar onset condition $19 \%$. A Friedman test revealed that there was no difference between these various conditions.

Initial saccades to the target

An ANOVA on latency for saccades correctly directed to the target showed that there was a significant main effect of condition $[F(2,22)=47.699, P<0.01]$. Planned comparisons showed that the mean saccade latency in the neutral condition (198 ms, SE $5 \mathrm{~ms}$ ) was significantly shorter than the mean saccade latency in the dissimilar onset condition [214 ms, SE $6 \mathrm{~ms} ; t(11)=5.670, P<0.01]$ and significantly shorter than the mean saccade latency in the similar onset condition [ $229 \mathrm{~ms}$, SE $5 \mathrm{~ms} ; t(11)=9.304, P<0.01$ ], suggesting that the distractor captured attention in both onset conditions. Furthermore, the mean saccade latency in the dissimilar onset condition was significantly shorter than the mean saccade latency in the similar onset condition $[t(11)=4.480, P<0.01]$. 
Time-course of correct saccades

To examine the effect of the two types of onset distractors on the latencies of saccades directed to the target, we calculated individual cumulative distribution functions of the saccade latencies for each subject. These were averaged in five bins using the vincentizing procedure (Ratcliff 1979). Figure 2 shows the distributions of the saccade latencies to the target in the three conditions. An ANOVA with conditions (neutral, dissimilar onset and similar onset) and bin as factors, revealed a main effect of condition $[F(2,22)=44.687, P<0.01]$. Planned comparisons revealed that in all bins, saccade latencies to the target in the neutral condition were significantly shorter than saccade latencies to the target in the dissimilar onset condition and the similar onset condition $(P<0.01)$. Furthermore, in all bins saccade latencies to the target in the dissimilar onset condition were significantly shorter than saccade latencies to the target in the similar onset condition $(P<0.05)$.

As is clear in Fig. 2, in addition to the effect of onset distractor, an effect of similarity of the distractor was found in all bins. The finding that the whole distribution in the similar onset condition is shifted relative to the distribution in the dissimilar onset condition, may be explained by assuming that once attention is captured by the distractor, it is more difficult to disengage attention from a similar distractor than from a dissimilar distractor.

Fixation duration on distractor

To determine whether the longer correct saccade latencies in the similar onset condition relative to the dissimilar onset condition may be explained in terms of disengagement of

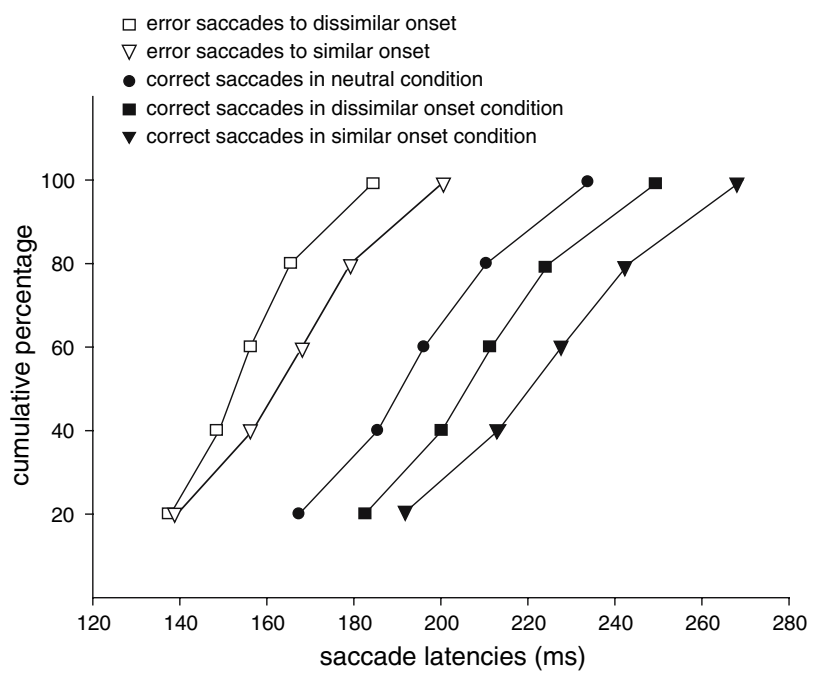

Fig. 2 Cumulative distribution functions of the latencies of the error saccades to the distractor in the dissimilar onset and the similar onset condition (open squares and triangles) and of the correct saccades in the neutral, the dissimilar onset and the similar onset condition (closed circles, squares and triangles) attention, we analyzed the fixation durations on the two types of onset distractors. Fixation durations included only those trials in which the error saccade was followed by a correct saccade to the target. Subjects (5) who had too few observations $(<10)$ of fixation durations given the above criteria were excluded from this analysis. Planned comparisons showed that mean fixation duration on the dissimilar distractor was shorter $(94 \mathrm{~ms}$, SE $8 \mathrm{~ms}$ ) than the mean fixation duration on the similar distractor $[128 \mathrm{~ms}$, SE $22 \mathrm{~ms}$; $t(6)=2.206, P<0.05$, one-tailed $]$.

Initial saccades to the distractor

Some of the subjects (4) had very few observations $(n<10)$ of saccades to the distractor in the dissimilar onset condition and were excluded from the following parametric analysis. In the dissimilar onset condition, mean saccade latency to the distractor $(158 \mathrm{~ms}$, SE $6 \mathrm{~ms})$ was significantly shorter than mean saccade latency to the target [213 ms, SE $9 \mathrm{~ms} ; t(7)=10.077, P<0.01]$. In addition, in the similar onset condition mean saccade latency to the distractor $(169 \mathrm{~ms}$, SE $5 \mathrm{~ms})$ was significantly shorter than mean saccade latency to the target $[229 \mathrm{~ms}$, SE $7 \mathrm{~ms}$; $t(7)=15.556, P<0.01]$. Importantly, the mean saccade latency to the dissimilar onset distractor was significantly shorter than the mean saccade latency to the similar onset distractor $[t(7)=3.600, P<0.01]$.

Time-course of error saccades

To examine the effect of the similarity of the distractors on saccade latencies to the distractor, we calculated individual cumulative distribution functions of the latencies. Figure 2 shows the distributions of the error saccades. An ANOVA with condition (dissimilar onset and similar onset) and bin as factors revealed a main effect of condition $[F(1,7)=12.383$, $P<0.01]$ and an interaction $[F(1.7,11.9)=7.439, P=0.01$, with Greenhouse-Geisser correction]. Furthermore, planned comparisons showed that the shortest saccade latencies in the first bin did not differ significantly $(P=0.62)$ between the two conditions and all other bins differed significantly $(P<0.05)$.

Initial saccades directed elsewhere than the target or distractor

In the neutral condition, all but one subject had very few observations $(n<10)$ of saccades directed to locations elsewhere than the target. Therefore, no parametric analysis was conducted for these saccade latencies. To conduct a $t$-test between the error saccades directed elsewhere than the target or distractor and the error saccades directed to a distractor, we excluded the same subjects (4) who had less than ten observations of error saccades directed to the dissimilar 
onset distractor. In the dissimilar onset condition, the mean latency of saccades directed elsewhere than the target or distractor (189 ms, SE $9 \mathrm{~ms}$ ) was significantly longer than mean latency of saccades directed to the distractor $[158 \mathrm{~ms}$, SE $6 \mathrm{~ms} ; t(7)=5.048, P<0.01]$. In the similar onset condition, the mean latency of saccades directed to a location elsewhere than the target or distractor (199 ms, SE $8 \mathrm{~ms}$ ) was significantly longer than mean latency of saccades directed to the distractor [169 ms, SE $5 \mathrm{~ms} ; t(7)=7.084$, $P<0.01]$. The shorter latencies of error saccades directed to the distractor relative to the error saccades directed elsewhere indicate that the distractor directed saccades were driven by the saliency information at that location.

\section{Discussion}

The results of Experiment 1 show that saccade latencies to the target are delayed by the presence of an additional distractor. This is consistent with the results obtained in a study by Godijn and Theeuwes (2002) (for similar finding see Theeuwes et al. 2003).

The phenomenon of increased saccade latencies in the presence of a remote distractor is called the remote distractor effect and is extensively investigated in a study by Walker et al. (1997) (see also Walker et al. 1995). They showed that the latencies of saccades to a visual target are increased when a distant onset distractor at a non-target location is presented simultaneously. In the present experiment, a similar distractor increased latencies of correct saccades more than a dissimilar distractor. The increase of correct saccade latencies in the presence of a similar distractor may be the result of slower attentional disengagement from a similar distractor. The fixation durations of error saccades to the distractors seem to corroborate this notion: fixation durations on a similar distractor were longer than fixation durations on a dissimilar distractor.

An important finding of the current experiment is that latencies of the saccades to the two types of distractors show a different time-course (see Fig. 2). Very early in time, the distributions do not differ, indicating that these saccades were driven purely on saliency information generated by the abrupt onset. Only later in time (after $\sim 150 \mathrm{~ms}$ ), the distributions begin to differentiate showing the influence of the similarity of the distractor. From these results we conclude that early in time, saliency information captures attention and the eyes automatically. Top-down goal processes initially do not prevent attentional capture by bottom-up saliency information, but rather becomes manifest later in time. Therefore, contingent capture may have a different time-course than automatic capture.

However, the results can also be interpreted from a conflict resolution point of view. Relative to the target, the sig- nal of the similar onset is a stronger competitor than the signal of the dissimilar onset. Due to the stronger competition in the similar onset condition, simple lateral inhibition (Ludwig et al. 2005; Usher and McClelland 2001) may account for the results. In this view, the conflict that arises between activity generated at the location of the target and at the location of the distractor would have taken extra time to resolve before the threshold is reached to execute a saccade.

To investigate whether the difference in saccade latencies was the result of processes resolving conflict or due to top-down processes driven by task-relevance of the distractor, we presented the onset in Experiment 2 either surrounding the target location (valid onset condition) or surrounding one of the non-target locations (invalid onset condition). As such, when the onset was presented at the target location, there was no conflict. In this way, the saliency information was either relevant for the task (when the onset was presented at the target location) or was irrelevant for the task (when the onset was presented at a non-target location). Figure $1 \mathrm{~b}$ shows the sequence of a trial in the valid onset condition. The conditions were blocked across trials and mixed with neutral (no-onset) trials. Thus, in one block, the onset, if present, always appeared at the target location while in the other block the onset always appeared at a non-target location. Given this set-up, the subjects knew in advance that either the onset was always valid (indicating consistently the target location) or always invalid (indicating consistently a distractor location). In the valid condition, conflict resolution cannot play a role because the task relevant salient signal is presented at the very same location as the target. In other words, there are no conflicting signals present in the visual field. If processes resolving conflict influenced the difference between the error saccade latencies in the different similarity conditions in Experiment 1, the saccade latencies to the valid onset should be shorter than the error saccade latencies to the invalid onset in this experiment. However, if top-down processes driven by task-relevance of the distractor caused the difference between the error saccades in Experiment 1, we should find similar saccade latency distributions in Experiment 2 as in Experiment 1.

\section{Experiment 2}

Method

\section{Participants}

Fourteen paid volunteers (aged 18-25) participated in the experiment. All participants had normal or corrected to normal vision. 


\section{Apparatus and design}

The same apparatus set-up as in Experiment 1 was used. Experiment 2 consisted of two blocks. One block with an onset at one of the non-target locations (invalid onset condition) mixed with no-onset trials (neutral condition) and one block with an onset at the target location (valid onset condition) mixed with no-onset trials (neutral condition). The block with the invalid onset condition consisted of 480 onset trials and 240 no-onset trials. The block with the valid onset condition consisted of 96 onset trials and 48 no-onset trials. Each block started with ten practice trials. The trials were randomly presented within a block and blocks were counterbalanced between participants.

We used the same paradigm as in Experiment 1 with the exception that in the onset conditions an open square, $3.05^{\circ}$ in diameter with a line-thickness of $0.2^{\circ}$, was presented surrounding one of the filled circles. The non-targets and the square were red and the target gray.

Each trial began with a white plus sign. After 1,400 ms the red circles were presented for at least $500 \mathrm{~ms}$ with an additional random jitter between 0 and $200 \mathrm{~ms}$. One of the red circles changed to gray indicating the target location. Simultaneously with the color change, the fixation point disappeared and in the onset trials, the square was presented surrounding one of the circles, either one of the nontargets (invalid onset condition) or the target (valid onset condition). The target was presented equally often at each of the four possible target locations: 1, 5, 7 or 11 o'clock. The onset was also presented either at 1, 5, 7 or 11 o'clock. In the valid onset condition, the onset was presented at target location and in the invalid onset condition at one of the other three remaining locations.

\section{Procedure}

The same as in Experiment 1 except that the feedback about their response time was displayed on the screen after every 24 th trial in both blocks.

\section{Results}

Of all trials the initial saccade latencies below $80 \mathrm{~ms}$ $(4.4 \%)$ and above $300 \mathrm{~ms}(3.7 \%)$ were excluded from analysis.

Initial saccades

In the neutral condition $95 \%$ of all initial saccades went to the target. In the invalid onset condition $65 \%$ of all initial saccades went to the target and in the valid onset condition $96 \%$.
A Friedman test on percentage correct saccades showed that there was a significant effect of the various conditions $\left[\chi^{2}(2, N=14)=23.286, P<0.01\right]$.

In the invalid onset condition $18 \%$ of all initial saccades went to the distractor. In the neutral condition $5 \%$ of all initial saccades went to locations elsewhere than the target and in the valid onset condition $4 \%$. In the invalid onset condition $17 \%$ went to locations elsewhere than the target or the distractor. A Friedman test on percentage saccades directed elsewhere showed a significant effect of condition $\left[\chi^{2}(2\right.$, $N=14)=23.286, P<0.01]$, indicating that a distractor present at a non-target location disturbed performance.

Initial saccades to the target

Saccade latencies to the target in the neutral condition did not differ significantly between the two blocks $(P=0.81)$, therefore the neutral condition trials in the two blocks were pooled. An ANOVA on saccade latency showed that there was a significant main effect of condition $[F(1.2$, $15.6)=41.514, P<0.01$, with Greenhouse-Geisser correction]. Planned comparisons showed that the mean saccade latency in the neutral condition (192 ms, SE $5 \mathrm{~ms}$ ) was significantly shorter than the mean saccade latency in the invalid onset condition [208 ms, SE $6 \mathrm{~ms}$; $t(13)=5.647$, $P<0.01]$. In addition, mean saccade latency in the valid onset condition (166 ms, SE $6 \mathrm{~ms}$ ) was significantly shorter than mean saccade latency in the neutral condition $[t(13)=5.982, P<0.01]$.

Time-course of correct saccades

To examine the effect of the relevance of the location of the onset on saccade latency, we calculated individual cumulative distribution functions of the saccade latencies to the target for each subject (see Fig. 3). An ANOVA with condition (neutral, valid onset and invalid onset) and bin as factors revealed a main effect of condition $[F(1.2,15.7)=40.707, P<0.01$, with Greenhouse-Geisser correction). Planned comparisons revealed that in all bins, saccade latencies to the target in the valid onset condition were significantly shorter than saccade latencies to the target in the neutral condition and the invalid onset condition $(P<0.01)$. Furthermore, in all bins saccade latencies to the target in the neutral condition were significantly shorter than saccade latencies to the target in the invalid onset location $(P<0.01)$.

Initial saccades to the valid and the invalid onset

To examine whether initial saccade latencies to the valid onset differed from initial saccade latencies to the invalid onset, we compared the mean saccade latencies. Mean 


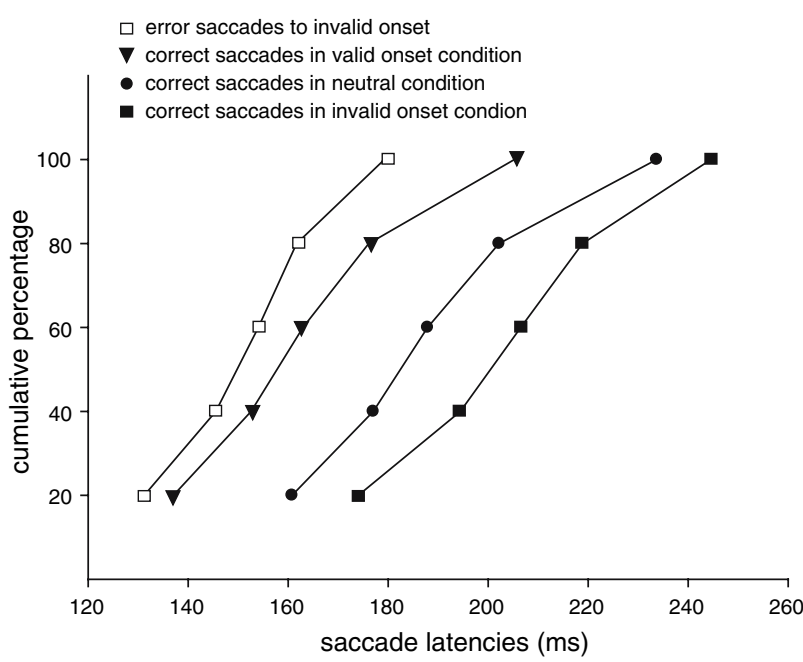

Fig. 3 Cumulative distribution functions of the latencies of the correct saccades in the valid onset, the neutral and the invalid onset condition (closed triangles, circles and squares) and of the error saccades to the onset in the invalid onset condition (open squares)

saccade latency to the invalid onset (error saccades) was significantly shorter (154 ms, SE $4 \mathrm{~ms}$ ) than mean saccade latency to the valid onset [166 ms, SE $6 \mathrm{~ms} ; t(13)=2.463$, $P<0.05]$.

Time-course of correct and error saccades

To examine the effect of the relevance of the onset on saccade latencies, we calculated individual cumulative distribution functions of the latencies of error saccades and correct saccades (see Fig. 3). An ANOVA with condition (valid onset and invalid onset) and bin as factors revealed a main effect of condition $[F(1,13)=5.890, P<0.05]$ and an interaction $[F(1.5,19)=18.374, \quad P<0.01$, with Greenhouse-Geisser correction]. Furthermore, planned comparisons showed that the saccades in the first three bins did not differ significantly. All other bins differed significantly $(P<0.05)$.

Initial saccades directed elsewhere than the target or invalid onset

In the neutral condition, there was no significant difference between the mean latency of saccades directed elsewhere than the target (185 ms, SE $5 \mathrm{~ms}$ ) and mean latency of saccades directed to the target (192 ms, SE $5 \mathrm{~ms}$ ). In the valid onset condition, all but one subject had very few observations $(n<10)$ of saccades directed to a location elsewhere than the target. Therefore, no parametric analysis was conducted for these saccade latencies. In the invalid onset condition, the mean latency of saccades directed elsewhere than the target or distractor (178 ms, SE $4 \mathrm{~ms}$ ) was significantly longer than mean latency of saccades directed to the invalid onset [154 ms, SE $4 \mathrm{~ms} ; t(13)=10.714, P<0.01$ ] The shorter latency of error saccades directed to the invalid onset relative to the error saccades directed elsewhere indicate that the onset directed saccades were driven by the saliency information at that location.

\section{Discussion}

The results of Experiment 2 show again that latencies of saccades to the target are delayed by the presence of an additional distractor (at a non-target location). The most revealing result of Experiment 2 concerns the time-course of the saccades in the valid onset condition. In this condition, both saliency information and task relevance information acted in favor of target selection. Note that the valid onset, if present, was presented at target location in $100 \%$ of time. Accordingly, subjects should have been fastest to select this location compared to any other locations in other conditions. However, subjects were fastest to select the invalid onset at a non-target location. This is consistent with Experiment 1 in which saccade latencies to a dissimilar distractor (task irrelevant) were shorter than saccade latencies to a similar distractor (task relevant).

As can be seen in Fig. 3, the latency distributions show a similar time-course as those observed in Experiment 1. The distribution of the error saccades to the invalid onset and the distribution of the correct saccades to the valid onset show that there is no difference between saccade latencies early in time, but later in time (after $\sim 160 \mathrm{~ms}$ ) the distributions start to differentiate. Whereas the information at target location was salient and relevant for the task, the information at a non-target location was salient but not relevant for the task. These results suggest that the difference between the distribution of correct saccades in the valid onset condition and the distribution of error saccades in the invalid onset condition may be the result of an interaction between bottom-up and top-down information. Importantly, the fact that these time-courses show the same pattern as in Experiment 1 indicates that the results of Experiment 1 cannot be attributed to processes resolving conflict. Whereas in Experiment 1, two signals at different locations would have to be resolved, in this experiment either two signals at one location (valid onset condition) or two signals at different locations (invalid onset condition) would have to be resolved. However, saccade latencies to the onset at a nontarget location (two signals at different locations) were shorter than saccade latencies to the onset at target location (two signals at the same location), suggesting that the interaction between bottom-up and top-down information affected these latter saccade latencies. 


\section{General discussion}

Results of the two experiments lead to the conclusion that automatic capture and contingent capture differ in timecourse. Early in time, capture was completely independent of task relevance, indicating that saliency information is processed first resulting in automatic capture. Later in time, task relevance influenced saccade latencies, indicating that task relevant information is processed later resulting in contingent capture.

The idea that bottom-up information can be influenced by top-down processes later in time would explain the difference between the obtained results in the additional distractor paradigm (Theeuwes 1992, 1994a, b; Yantis and Jonides 1984, 1990) and the distractor cueing in advance studies (Folk et al. 1992, 1994). In the first $150 \mathrm{~ms}$, saliency information guides attentional selection and after $150 \mathrm{~ms}$ slower additional top-down processes become available. As a result, a distractor that is contingent on task demands will be more difficult to inhibit only later in time. In addition, the results of Experiment 2 showed that a top-down search strategy for singletons (Bacon and Egeth 1994) is not sufficient to explain attentional capture. In the valid onset condition, the onset at target location is the only unique singleton in the display. If participants had adopted a search strategy for singletons, saccade latencies to the valid onset at the target location should have been shorter than saccade latencies to an invalid onset at a non-target location. However, we found that saccade latencies to a singleton (invalid onset) in a display with another singleton (the target) were shorter than saccade latencies to one unique singleton (valid onset) in the display. Accordingly, these results suggest that a top-down strategy for unique singletons, even when appropriate as in our valid onset condition, does not necessarily speed up selection but may in fact increase saccade latencies.

Although our results are consistent with the notion that saccade latencies driven by saliency are shorter than saccades driven by top-down goals (e.g., Godijn and Theeuwes 2002; van Zoest and Donk 2005; van Zoest et al. 2004), the current findings seem to suggest an integration of saliency and top-down information. In contrast to earlier conclusions of van Zoest and colleagues (van Zoest and Donk 2005; van Zoest et al. 2004) who claimed that saliency and top-down processes operate in different and independent time-windows, our findings imply that topdown processes integrate with saliency information (see also Godijn and Theeuwes 2002; Ludwig and Gilchrist 2002, 2003a, b). This is consistent with the competitive integration model (Godijn and Theeuwes 2002). This functional model states that saccades are generated in a common spatiotopic saccade map in which bottom-up and top-down information is integrated (see also Ludwig and
Gilchrist 2002, 2003a, b). If a location in the saccade map is activated, neighboring locations will also get activated but due to lateral inhibition, more distant locations will be inhibited. In addition, inhibition can also be achieved through an additional top-down mechanism that directly affects the activation within the saccade map (e.g., Tipper et al. 2001). If the activation in the map reaches a certain threshold, a saccade to that location is executed. Results from studies with the additional distractor paradigm (Godijn and Theeuwes 2002) suggested that bottom-up saliency information activates the saccade map first before top-down information is available. Godijn and Theeuwes (2002) concluded this on the basis of several findings. First, error saccades to a salient distractor were faster than correct saccades to the target and second, the location of the target in relation to the location of the distractor had no effect on the fastest saccades while it did have an effect on the slower saccades. They argued that top-down information reaches the saccade map later because additional processing is necessary for goal-directed saccades in order to determine if the properties of the stimulus are related to the goal.

The competitive integration model is supported by the neurophysiology of saccade programming. Godijn and Theeuwes placed their model in the superior colliculus (SC) which plays an essential role in saccade programming (for reviews, see Schall 1995; Van der Stigchel et al. 2006) and is assumed to integrate top-down activation and saliency activation from the environment (Trappenberg et al. 2001). The SC receives top-down activations from the frontal eye fields (FEF), supplementary eye fields, the lateral intraparietal area and the dorsolateral prefrontal cortex. Integration of bottom-up and top-down information in the process of target selection, however, is not only observed in the SC but also in other areas involved in oculomotor programming such as the FEF (see for example Everling and Munoz 2000; for review Schall 1995).

Whereas Godijn and Theeuwes (2002) argue that bottom-up and top-down control are integrated in a common saccade map, Irwin et al. (2000) argued that the two different selection mechanisms are each controlled by different parallel pathways: one cortical pathway, responsible for voluntary goal-directed saccades and one sub-cortical pathway depending on the $\mathrm{SC}$ and responsible for reflexive involuntary saccades (e.g., Schall 1995). They reached this conclusion after they determined which salient items induced oculomotor capture. Abrupt onsets, luminance increments and color changes were used as the salient distractor. Results showed that abrupt onsets and luminance increment of an irrelevant distractor captured the eyes. A color change of the distractor, however, did not have these effects. Based on the idea that neurons in the SC appear not to discriminate color (Marrocco and Li 1977), Irwin et al. 
argued that the color change in their experiment could never elicit a reflexive like saccade. However, although the initial activity in the SC does not reflect color discrimination, target identity information on the basis of color does reach the SC $100 \mathrm{~ms}$ after stimulus onset (McPeek and Keller 2002, 2004).

The hypothesis that involuntary saccades based on saliency are processed by a different pathway than goaldirected saccades (e.g., Irwin et al. 2000; Schall 1995) seems not consistent with our results. However, we cannot completely rule out the idea that the generation of saliencydriven saccades is processed by a sub-cortical route. In this study, bottom-up saliency information is processed faster than top-down color information. Nevertheless, our results seem to contradict a clear dissociation between a sub-cortical pathway responsible for involuntary saccades and a cortical pathway responsible for voluntary saccades. In this study, top-down activation seems to enhance bottom-up saliency information. The integration of information is not only present in the error saccades to a similar salient distractor, but also shown by the shorter correct saccade latencies to the salient valid onset relative to correct saccade latencies in the baseline condition when no onset is presented. However, integration of top-down and bottom-up information also seems to slow the oculomotor system. Saccade latencies to the similar and valid onset were longer than saccade latencies to a dissimilar or invalid onset. Especially, experiment 2 reveals that top-down goal information could decelerate instead of accelerate the oculomotor system. Subjects in this experiment had no reason to inhibit the saliency information at the target location because it was $100 \%$ valid. Nevertheless, the saccade latency distributions showed that saccades were slowed by this saliency information (for similar results see van Zoest and Donk 2005). Initial automatic inhibition of saliency information could explain these results. Kramer et al. (2005), already speculated in addition to an intentional type of inhibition about an automatic type of inhibition. However, how and where these two types of inhibition are controlled is not yet clear. Future research about automatic and contingent capture could give more insight into the processes involved in the activation and inhibition of saliency information and topdown signals acting upon this information, whether inhibitory or excitatory.

Acknowledgments We would like to thank Casimir Ludwig, Raymond Klein and Joshua Salmon for comments on earlier versions of this paper. This research was funded by a grant from Human Frontier Science Program (HFSP-RGP39/2005) to Jan Theeuwes.

Open Access This article is distributed under the terms of the Creative Commons Attribution Noncommercial License which permits any noncommercial use, distribution, and reproduction in any medium, provided the original author(s) and source are credited.

\section{References}

Bacon WF, Egeth HE (1994) Overriding stimulus-driven attentional capture. Percept Psychophys 55(5):485-496

Everling S, Munoz DP (2000) Neuronal correlates for preparatory set associated with pro-saccades and anti-saccades in the primate frontal eye field. J Neurosci 20(1):387-400

Folk CL, Remington RW, Johnston JC (1992) Involuntary covert orienting is contingent on attentional control settings. J Exp Psychol Hum Percept Perform 18(4):1030-1044

Folk CL, Remington RW, Wright JH (1994) The structure of attention control: contingent attentional capture by apparent motion, abrupt onset, and color. J Exp Psychol Hum Percept Perform 20(2):317329

Godijn R, Theeuwes J (2002) Programming of endogenous and exogenous saccades: evidence for a competitive integration model. J Exp Psychol Hum Percept Perform 28(5):1039-1054

Irwin DE, Colcombe AM, Kramer AF, Hahn S (2000) Attentional and oculomotor capture by onset, luminance and color singletons. Vision Res 40(10-12):1443-1458

Kramer AF, de Sather J, Cassavaugh ND (2005) Development of attentional and oculomotor control. Dev Psychol 41(5):760-772

Ludwig CJH, Gilchrist ID (2002) Stimulus-driven and goal-driven control over visual selection. J Exp Psychol Hum Percept Perform 28(4):902-912

Ludwig CJH, Gilchrist ID (2003a) Goal-driven modulation of oculomotor capture. Percept Psychophys 65(8):1243-1251

Ludwig CJH, Gilchrist ID (2003b) Target similarity affects saccade curvature away from irrelevant onsets. Exp Brain Res 152(1):60 69

Ludwig CJH, Gilchrist ID, McSorley E (2005) The remote distractor effect in saccade programming: channel interactions and lateral inhibition. Vision Res 45:1177-1190

Marrocco RT, Li RH (1977) Monkey superior colliculus: properties of single cells and their afferent inputs. J Neurophysiol 40(4):844860

McPeek RM, Keller EL (2002) Saccade target selection in the superior colliculus during a visual search task. J Neurophysiol 88:20192034

McPeek RM, Keller EL (2004) Deficits in saccade target selection after inactivation of superior colliculus. Nat Neurosci 7:757-763

Munoz DP, Dorris MC, Pare M, Everling S (2000) On your mark, get set: brainstem circuitry underlying saccadic initiation. Can J Physiol Pharmacol 78(11):934-944

Ratcliff R (1979) Group reaction-time distributions and an analysis of distribution statistics. Psychol Bull 86(3):446-461

Schall JD (1995) Neural basis of saccade target selection. Rev Neurosci 6(1):63-85

Theeuwes J (1992) Perceptual selectivity for color and form. Percept Psychophys 51(6):599-606

Theeuwes J (1994a) Stimulus-driven capture and attentional set: selective search for color and visual abrupt onsets. J Exp Psychol Hum Percept Perform 20(4):799-806

Theeuwes J (1994b) Endogenous and exogenous control of visual selection. Perception 23(4):429-440

Theeuwes J (1995) Abrupt luminance change pops-out; abrupt color change does not. Percept Psychophys 57:637-644

Theeuwes J (2004) Top-down search strategies cannot override attentional capture. Psychon Bull Rev 11(1):65-70

Theeuwes J, Kramer AF, Hahn S, Irwin DE (1998) Our eyes do not always go where we want them to go: capture of the eyes by new objects. Psychol Sci 9(5):379-385

Theeuwes J, de Vries GJ, Godijn R (2003) Attentional and oculomotor capture with static singletons. Percept Psychophys 65(5):735-746 
Tipper SP, Howard LA, Paul MA (2001) Reaching affects saccade trajectories. Exp Brain Res 136(2):241-249

Trappenberg TP, Dorris MC, Munoz DP, Klein RM (2001) A model of saccade initiation based on the competitive integration of exogenous and endogenous signals in the superior colliculus. J Cogn Neurosci 13(2):256-271

Usher M, McClelland JL (2001) The time course of perceptual choice: the leaky, competing accumulator model. Psychol Rev 108(3):550-592

Van der Stigchel S, Theeuwes J (2006) Our eyes deviate away from a location where a distractor is expected to appear. Exp Brain Res 169(3):338-349

Van der Stigchel S, Meeter M, Theeuwes J (2006) Eye movement trajectories and what they tell us. Neurosci Biobehav Rev 30(5):666-679

van Zoest W, Donk M (2005) The effects of salience on saccadic target selection. Vis Cogn 12(2):353-375 van Zoest W, Donk M (2006) Saccadic target selection as a function of time. Spat Vis 19(1):61-76

van Zoest W, Donk M, Theeuwes J (2004) The role of stimulus-driven and goal-driven control in saccadic visual selection. J Exp Psychol Hum Percept Perform 30(4):746-759

Walker R, Kentridge RW, Findlay JM (1995) Independent contributions of the orienting of attention, fixation offset and bilateral stimulation on human saccadic latencies. Exp Brain Res 103(2):294-310

Walker R, Deubel H, Schneider WX, Findlay JM (1997) Effect of remote distractors on saccade programming: evidence for an extended fixation zone. J Neurophysiol 78(2):1108-1119

Yantis S, Jonides J (1984) Abrupt visual onsets and selective attention: evidence from visual search. J Exp Psychol Hum Percept Perform 10(5):601-621

Yantis S, Jonides J (1990) Abrupt visual onsets and selective attention: voluntary versus automatic allocation. J Exp Psychol Hum Percept Perform 16(1):121-134 